PHYSICAL REVIEW D 92, 109902(E) (2015)

\title{
Erratum: Bounds on QCD axion mass and primordial magnetic field from cosmic microwave background $\mu$ distortion [Phys. Rev. D 90, 123527 (2014)]
}

\author{
Damian Ejlli \\ (Received 27 September 2015; published 2 November 2015)
}

DOI: 10.1103/PhysRevD.92.109902

PACS numbers: 95.35.+d, 14.80.Va, 98.62.En, 98.70.Vc, 99.10.Cd

It has come to my attention that there are some inequality typos and numerical errors in the abstract and text that may give a misleading interpretation to the obtained results, especially in connection with the limit on the axion mass. Consequently, in the abstract the following corrections have been made:

(1) In the line number 4 the corrected limit for the axion mass (by using the upper limit on the magnetic field strength $B \lesssim 3.2 \mathrm{nG})$ is $m_{a} \lesssim 4.8 \times 10^{-5} \mathrm{eV}$.

(2) In the line number 6 the corrected value obtained for the magnetic field strength in the case of the KSVZ axion model is $B \simeq 46 \mathrm{nG}$.

(3) In the line number 7 the corrected value obtained for the magnetic field strength in the case of the DFSZ axion model is $B \simeq 130 \mathrm{nG}$.

The same changes made in the abstract have also been made in the text. Therefore the changes made in the text are as follows:

(4) On page number 4 , first column and line number 25 , the corrected value for the limit on the axion mass is $m_{a} \lesssim 4.8 \times 10^{-5} \mathrm{eV}$.

(5) On page number 4, second column and last line from the top, the corrected value for the magnetic field strength for the $\mathrm{KSVZ}$ axion model is $B \simeq 46 \mathrm{nG}$.

(6) On page number 5, first column and line number 1 the corrected value for the magnetic field strength for the DFSZ axion model is $B \simeq 130 \mathrm{nG}$.

The changes made to the numerical values do not affect Figs. 1 and 2 whatsoever. 